\title{
Communications
}

\section{Le contrôle de qualité des vaccins et de la chaîne du froid lors des campagnes nationales de vaccination}

E. Couacy-Hymann '

A. Kodjo ${ }^{2}$

M. Ouattara ${ }^{3}$

K. Kanga ${ }^{3}$

S. Diawara ${ }^{2}$

\section{J. Domenech ${ }^{4}$}

COUACY-HYMANN (E.), KODJO (A.), OUATTARA (M.), KANGA (K.), DIAWARA (S.), DOMENECH (J.). Le contrôle de qualité des vaccins et de la chaîne du froid lors des campagnes nationales de vaccination. Revue Élev. Méd. vét. Pays trop., 1992, 45 (2) : 129-133

Durant les deux campagnes de vaccination contre la peste bovine et la péripneumonie contagieuse bovine en 1989 et 1990, 507 échantillons de vaccin ont été prélevés tout le long de la chaîne de distribution qui va de la Pharmacie vétérinaire d'Abidjan au vaccinateur sur le terrain, afin de contrôler la qualité de la suspension vaccinale que reçoit l'animal. Parallèlement, la qualité de la chaîne du froid était vérifięe. Au total 463 titrages ont été réalisés. Aucun titre ne s'est révélé en dessous des normes de l'OIE/FAO, à savoir $10^{2,5} \mathrm{DICT}_{50} / \mathrm{ml}$ pour la valence peste bovine et $10^{7}$ germes viables $/ \mathrm{ml}$ pour la valence péripncumonique. Mots clés: Bovin - Vaccin - Peste bovine - Péripneumonie contagieuse bovine Stockage au froid - Transport frigorifique - Côte-d'Ivoire.

\section{Introduction}

En 1987, un échantillon de vaccin antibovipestique a été prélevé avant la campagne de vaccination pour un contrôle du titre. En effet, l'aspect physique de la pastille de vaccin lyophilisé était "suspect". Le titrage avait donné $10^{1,5} \mathrm{DICT}_{50} / \mathrm{ml}(1)$.

Partant de ce constat, et avec l'exécution du projet PARC $^{*}$, une unité de contrôle de qualité des vaccins a été mise en place au Laboratoire de Bingerville en 1988. Ainsi, en Côte-d'lvoire, en plus du travail accompli en amont par l'unité de contrôle de Dakar (PANVAC ${ }^{\star \star}$ ), une surveillance continue de la qualité des vaccins utilisés est menée durant les campagnes annuelles de vaccination.

1. Laboratoire central de pathologie animale, BP 206, Bingerville. Côte-d'lvoire.

2. Laboratoire régional de pathologie animale, BP 32, Korhogo, Côte-d'lvoire.

3. Laboratoire régional de pathologie animale, $01 \mathrm{BP} 1366$, Bouaké, Côte-d'Ivoire.

4. OUA-IBAR, POB 30786, Nairobi, Kenya.

Reçu le 26.11.91, accepté le 9.6.1992.

* PARC : Pan African Rinderpest Campaign. OUA-IBAR/CEE, Nairobi, Kenya.

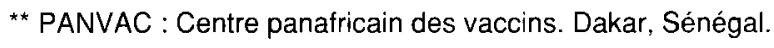

La présente étude concerne celles de 1989 et 1990.

\section{Matériel et méthode}

\section{Prélèvement de flacons de vaccin}

A l'arrivée de chaque lot de vaccin, un échantillon est envoyé au Laboratoire central de pathologie animale de Bingerville (LPA) par la Pharmacie vétérinaire en vue d'un contrôle de la qualité avant l'usage sur le terrain. Ainsi, ont été éprouvés des échantillons provenant de 10 lots de vaccin monovalent peste bovine (PB) ( 7 lots en 1989 et 3 en 1990), 15 lots de vaccin bivalent PB et péripneumonie contagieuse bovine (PPCB) (9 lots en 1989 et 6 en 1990) et un lot de vaccin monovalent PPCB en 1990.

Pendant les deux campagnes de vaccination, les équipes des laboratoires, indépendantes des équipes de vaccination, ont sillonné toutes les régions du pays pour prélever des échantillons de vaccin si possible sur les lieux de vaccination en prenant soin de ne pas prévenir les vaccinateurs de cette visite afin de mieux les apprécier au travail. Au total 507 flacons ont été prélevés (tabl. I).

Contrôle de la chaîne du froid : la chaîne de distribution des vaccins va de la Pharmacie vétérinaire d'Abidjan $(\mathrm{Cl})$ aux zones d'encadrement, puis aux secteurs et enfin aux centres d'encadrement. Le ravitaillement des zones à partir de la Pharmacie se fait par un véhicule frigorifique. Les secteurs reçoivent les vaccins à partir des zones dans des glacières contenants des réfrigérants. Enfin, les centres sont alimentés à partir des secteurs toujours à l'aide de glacières.

Les zones d'encadrement, les secteurs et certains centres possèdent des congélateurs et des réfrigérateurs électriques. D'autres centres reculés ont des réfrigérateurs à pétrole. Cependant, quelques uns, ne possédant pas de système de froid dynamique, ont des glacières et sont ravitaillés en barres de glace deux à trois fois par semaine.

Les fermes à visiter sont prévenues la veille, et le vaccinateur emporte chaque jour dans une glacière le nombre de flacons de vaccin nécessaire. Sur le lieu de vaccina-

TABLEAU I Nombre de flacons de vaccin prélevés durant les deux campagnes de vaccination.

\begin{tabular}{|c|c|c|c|c|}
\hline \multirow{2}{*}{ Année } & \multirow{2}{*}{$\begin{array}{c}\text { Vaccin bivalent } \\
\text { PB-PPCB }\end{array}$} & \multicolumn{2}{|c|}{ Vaccin monovalent } & \multirow{2}{*}{ Total } \\
\cline { 2 - 4 } & & PB & PPCB & \\
\hline 1989 & 270 & 58 & 0 & 328 \\
\hline 1990 & 140 & 36 & 3 & 179 \\
\hline Total & 410 & 94 & 3 & 507 \\
\hline
\end{tabular}


tion, la seringue, entourée d'un linge humide, est remise dans la glacière à chaque temps mort.

Le contrôle de la qualité de la chaîne du froid consiste à vérifier le bon état de fonctionnement des congélateurs et réfrigérateurs, la présence permanente de glace dans les glacières, la bonne pratique du vaccinateur au travail qui doit, notamment, constater l'état de la pastille de vaccin lyophilisé et rejeter le reste du vaccin reconstitué à la fin de chaque demi-journée. Le prélèvement des échantillons de vaccin a lieu, si possible, à tous les maillons de la chaîne du froid.

La solution molaire de sulfate de magnésium constitue le principal diluant utilisé. Cependant, certaines régions forestières ont été ravitaillées en eau physiologique par le Laboratoire de pathologie animale.

\section{Analyses des échantillons prélevés}

Les échantillons envoyés par la Pharmacie vétérinaire subissent les tests suivants : titrage, stérilité (vaccins viraux), pureté (virus bactériens), contrôle du vide, innocuité sur souris. Les flacons de vaccin prélevés sur le terrain subissent les mêmes tests à l'exception de celui d'innocuité sur souris.

- Technique de titrage de la valence PB : on a utilisé la méthode en plaque de 96 trous $(5,7)$ avec des cellules Vero. On élimine la valence PPCB des vaccins bivalents en ajoutant $1 \mathrm{ml}$ de Suanovil 20 ND pour 100 doses.

- Technique de titrage de la valence PPCB, selon la méthode en tubes $(10)$ : on a utilisé pour la galerie secondaire $(5$ tubes par dilution à partir de la dilution primaire $10^{-7}$ ) le milieu de Gourlay au rouge de phénol (10).

- Test de pureté : on réalise une coloration de Gram et de Giemsa sur le contenu d'un flacon de vaccin après sa reconstitution.

- Test de stérilité : différents milieux de culture bactériologique sont ensemencés avec la suspension vaccinale :

* bouillon tryptose-sérum ... 5 tubes

* tryptose agar-sérum ... 2 boîtes de Petri

* bouillon au thioglycolate ... 5 tubes

* milieu de Sabouraud ... 2 boîtes de Petri

L'incubation se fait à $37^{\circ} \mathrm{C}$ pendant 48 à $72 \mathrm{~h}$.

- Contrôle du vide : il s'effectue à l'aide d'un appareil* qui émet une lumière violette quand on applique l'électrode sur un flacon de vaccin ayant un vide convenable.

- Test d'innocuité sur souris : cinq souris adultes sont inoculées par voie intrapéritonéale avec $0,5 \mathrm{ml}$ de la suspen-

*EDWARDS ST4 M Spark Tester. sion vaccinale; ces animaux inoculés sont gardés en observation pendant trois semaines. Deux souris non éprouvées sont prises comme témoin.

- Calcul des titres de vaccin : le titre de la valence PB est déterminé par la méthode de Spermann-Kärber ; celui de la valence PPCB est fait par la méthode du "Most Probable Number" (8).

\section{Résultats}

\section{Titrage des valences $P B$ et $P P C B$}

Au cours des deux campagnes de vaccination 463 titrages ont été effectués pour 507 flacons récoltés (44 (507-463) flacons étaient en double), soit 274 titrages pour la valence PB et 189 pour la valence PPCB. Aucun titre ne s'est révélé en dessous des normes de l'OIE/FAO, c'est-à-dire $10^{2,5} \mathrm{DICT}_{50} / \mathrm{ml}$ pour la valence PB et $10^{7}$ germes viables $/ \mathrm{ml}$ pour la valence $\operatorname{PPCB}(7,10)$ sur le terrain (tabl. II, III).

\section{Test de pureté et de stérilité}

Toutes les colorations de Gram et de Giemsa réalisées sur la suspension vaccinale de chaque flacon ont montré la pureté des différents lots de vaccin pour la valence PPCB. De même, les milieux de culture ensemencés sont restés négatifs, prouvant la stérilité des vaccins pour la valence PB.

\section{Test d'innocuité sur souris}

Toutes les souris inoculées avec la suspension vaccinale sont restées vivantes prouvant ainsi l'innocuité des lots de vaccin reçus.

\section{Contrôle du vide à l'intérieur du flacon}

Tous les flacons de vaccin contrôlés étaient sous vide.

\section{Discussion}

Les deux campagnes de vaccination de 1989 et 1990 ont permis de réaliser 463 titrages et de mener parallèlement une surveillance de la chaîne du froid. Aucun dysfonctionnement dans la chaîne et aucun titre en dessous des normes recommandées pour les deux valences (PB, PPCB) n'ont été observés.

Un échantillon de chaque lot de vaccin reçu à la Pharmacie vétérinaire a été envoyé pour contrôle au LPA de Bingerville. Le même lot retrouvé en bout de chaîne, sur le terrain (au niveau des parcs de vaccination) a fait l'objet d'un titrage au LPA. En revanche, il n'a pas été toujours possible de suivre un lot à tous les stades intermédiaires de distribution (zone et secteur). Il est en fait surtout intéressant de comparer le titre du vaccin à son arrivée à la Pharmacie vétérinaire et sur le terrain. Cette comparaison a fait défaut pour cinq lots qui n'ont pas pu être retrouvés sur le terrain. Cet écueil est dû au décollement des étiquettes des flacons. En effet, le but de ce contrôle est avant tout de démontrer la qualité du vaccin au moment de son injection à l'animal. 
TABLEAU II Résultats obtenus sur 274 titrages de la valence PB $\left(D I C T_{s 0} /\right.$ ml exprimés en $\left.\log _{t 0}\right)$.

\begin{tabular}{|c|c|c|c|c|c|c|}
\hline $\begin{array}{c}\text { Type } \\
\text { Vaccin }\end{array}$ & $\begin{array}{c}\text { Numéro } \\
\text { Lot }\end{array}$ & Ph. vét. * & Zone ** & Secteur ** & Terrain ** & Différence \\
\hline Monovalent & $\begin{array}{c}15 \\
21 \\
82 \\
230 \\
30 \\
237 \\
234 \\
363 \\
31 \\
32 \\
\end{array}$ & $\begin{array}{c}3,5 \\
3,5 \\
3,9 \\
4,68 \\
3,5 \\
3,9 \\
4,1 \\
4,1 \\
3,5 \\
2,5 \\
\end{array}$ & $\begin{array}{l}3,1-4,0 \\
2,6-3,2 \\
4,0-4,6 \\
3,0-4,0 \\
3,2-3,8 \\
3,8-4,4\end{array}$ & $\begin{array}{l}3,9-4,4 \\
3,3-3,9\end{array}$ & $\begin{array}{l}2,7-3,1 \\
2,8-3,2 \\
3,0-3,5 \\
3,5-4,5 \\
3,3-3,5 \\
3,1-3,7 \\
3,9-4,1 \\
3,5-3,9\end{array}$ & $\begin{array}{c}0,4-0,8 \\
0,3-0,7 \\
0,5-0,9 \\
0,18-1,18 \\
0,0-0,2 \\
0,2-0,8 \\
0,0-0,2 \\
0,2-0,6\end{array}$ \\
\hline Bivalent & $\begin{array}{l}342 \\
345 \\
364 \\
255 \\
259 \\
268 \\
269 \\
270 \\
272 \\
273 \\
341 \\
348 \\
356 \\
260 \\
264\end{array}$ & $\begin{array}{c}4,1 \\
3,9 \\
4,3 \\
4,5 \\
4,16 \\
4,37 \\
4,16 \\
4,5 \\
3,8 \\
4,0 \\
4,1 \\
4,5 \\
4,5 \\
4,1 \\
3,9\end{array}$ & $\begin{array}{l}3,7-4,2 \\
3,7-4,5 \\
3,2-4,2 \\
3,6-4,2 \\
3,2-4,2 \\
3,7-4,0 \\
3,8-4,3\end{array}$ & $\begin{array}{l}3,7-3,9 \\
3,7-4,1 \\
3,1-3,8 \\
3,6-4,5 \\
3,5-4,3 \\
\\
3,9-4,9 \\
3,4-4,0 \\
3,5-4,0\end{array}$ & $\begin{array}{l}3,7-4,1 \\
3,1-3,9 \\
3,1-4,1 \\
3,7-4,1 \\
3,3-3,9 \\
3,3-4,1 \\
3,3-3,9 \\
\\
3,4-3,9 \\
3,3-4,0 \\
3,3-3,9 \\
\\
3,7-4,1 \\
3,3-3,9\end{array}$ & $\begin{array}{c}0,0-0,4 \\
0,0-0,8 \\
0,2-1,2 \\
0,4-0,8 \\
0,26-0,86 \\
0,27-1,07 \\
0,26-0,86 \\
\\
0,0-0,4 \\
0,0-0,7 \\
0,2-0,8\end{array}$ \\
\hline
\end{tabular}

Intervalle de confiance : titre indiqué $\pm 0,5$ pour 5 cupules par dilution (9).

* : Pharmacie centrale vétérinaire (Abidjan).

** : Les valeurs indiquées représentent la borne inférieure et la borne supérieure de l'intervalle pour chaque série de titrages.

TABLEAU III Résultats obtenus sur 189 numérations de la valence PPCB (MPN/ml exprimés en log $\left.{ }_{r}\right)$.

\begin{tabular}{|c|c|c|c|c|c|c|}
\hline $\begin{array}{c}\text { Type } \\
\text { Vaccin }\end{array}$ & $\mathrm{N}^{\circ}$ Lot & Ph. vét. * & Zone ${ }^{\star \star}$ & Secteur ** & Terrain ** & Différence \\
\hline Monovalent & 232 & 7,23 & & & & \\
\hline \multirow{15}{*}{ Bivalent } & 268 & 7,54 & $7,46-7,49$ & $7,17-7,47$ & $7,04-7,97$ & $0,43-0,5$ \\
\hline & 269 & 7,54 & $7,53-7,55$ & $7,25-7,58$ & $7,0-7,63$ & $0,09-0,54$ \\
\hline & 270 & 7,96 & & $7,93-7,99$ & & \\
\hline & 272 & 7,96 & $7,88-7,92$ & $7,30-7,60$ & $7,11-7,89$ & $0,07-0,85$ \\
\hline & 273 & 7,96 & $7,32-7,38$ & $7,25-7,39$ & $7,11-7,89$ & $0,07-0,85$ \\
\hline & 260 & 7,34 & $7,25-7,36$ & & $7,10-7,34$ & $0,0-0,24$ \\
\hline & 264 & 7,30 & $7,17-7,48$ & & $7,23-7,32$ & $0,02-0,07$ \\
\hline & 342 & 7,54 & $7,46-7,82$ & $7,36-7,7$ & $7,23-7,89$ & $0,31-0,35$ \\
\hline & 345 & 7,54 & & $7,47-7,98$ & $7,14-7,89$ & $0,35-0,4$ \\
\hline & 364 & 7,54 & & $7,47-7,60$ & $7,23-7,84$ & $0,30-0,31$ \\
\hline & 255 & 7,44 & $7,41-7,47$ & $7,25-7,76$ & $7,11-7,89$ & $0,33-0,66$ \\
\hline & 341 & 7,23 & & & $7,23-7,89$ & $0,0-0,66$ \\
\hline & 348 & 7,14 & & & & \\
\hline & 356 & 7,63 & & & & \\
\hline & 259 & 7,30 & $7,23-7,30$ & & $7,04-7,89$ & $0,26-0,59$ \\
\hline
\end{tabular}

Intervalle de confiance : MPN $\pm 0,5$ pour 5 tubes par dilution (en dilution secondaire) (4).

: idem tableau II; ** : idem tableau III. 
Les tableaux II et III présentent les résultats des 463 titrages. Les résultats de la colonne "Ph. vét." sont issus d'un seul titrage par flacon. Ceux des colonnes: "zone" et "secteur", sont obtenus à partir de trois et d'environ cinq titrages du même lot (1 titrage/flacon), respectivement. Enfin, les résultats de la colonne "terrain" sont également obtenus à partir d'environ huit titrages du même lot (1 titrage/flacon). La colonne "différence" indique la plus faible chute de titre et la plus forte pour une série de titrages effectués sur un même lot: chaque borne de l'intervalle de la colonne "terrain" est comparée au titre à l'arrivée.

\section{Valence $P B$}

Les 274 titrages effectués n'ont donné aucun titre en dessous de $10^{2,5} \mathrm{DICT}_{50} / \mathrm{ml}$ au moment de l'injection à l'animal. Néanmoins, il a été remarqué une légère chute du titre entre l'arrivée à la Pharmacie vétérinaire (Abidjan) et le terrain. Cette chute peut aller de 00 à $1,20 \log _{10}$ avec seulement trois cas de chute du titre supérieure à $1 \log _{10}$. Signalons par ailleurs que le protocole de titrage (1 titrage/flacon) ne permet pas de donner une valeur statistique formelle à ces comparaisons de titre : le seul résultat patent et indiscutable est que tous les lots restent au dessus des normes admises. Ceci démontre l'excellente qualité de la chaîne du froid en Côte-d'Ivoire.

\section{Valence $P P C B$}

Cent quatre vingt neuf numérations ont été faites pour cette valence, à partir de 16 lots de vaccin différents; aucune ne s'est révélée inférieure à la norme de $10^{7}$ germes viables $/ \mathrm{ml}$. Si les résultats obtenus sont issus d'une mesure par échantillon de vaccin prélevé en un lieu donné, ce sont, en revanche, huit titrages environ qui ont été réalisés par lot de vaccin (au niveau du parc de vaccination). On préconise habituellement plusieurs mesures en tubes ou en plaque de 96 trous (9) afin de pallier les irrégularités observées dans les résultats. Ces irrégularités seraient dues à la présence d'agglutinats de germes qui rendent les suspensions vaccinales hétérogènes (3) (PERREAU, communication personnelle), mais aussi à la méthode elle-même (9). La multiplication des numérations en tubes aurait alourdi considérablement l'étude. Comme pour la $\mathrm{PB}$, on a comparé les titres à l'arrivée à la Pharmacie vétérinaire et sur le terrain pour un même lot. La chute du titre des vaccins a été faible variant entre 00 et $0,85 \log _{10}$ germes. Tous les flacons ont gardé un titre supérieur à $7 \log _{10}$ germes viables $/ \mathrm{ml}$. Ceci confirme une fois de plus la qualité de la chaîne du froid. Rappelons que la conservation du vaccin PPCB lyophilisé a été étudiée par divers auteurs. Ainsi, pour la souche KH3J, la perte de $1 \log _{10}$ est atteinte en 9 à 16 semaines si on place le vaccin à $28^{\circ} \mathrm{C}, 6$ à 14 semaines à $37^{\circ} \mathrm{C}$ et 12 à 14 jours à $46{ }^{\circ} \mathrm{C}(3)$. Quand on compare ces températures à celles constatées tout le long de la chaîne du froid en Côte-d'Ivoire et même si la souche vaccinale est ici différente (souche T1-SR et non KH3J), on ne peut qu'aboutir à d'excellents résultats sur le terrain. II faut noter cependant que dans la conservation d'un vaccin, l'humidité résiduelle joue un rôle très important. Ce point n'a pas fait l'objet de cette étude.

\section{Conclusion}

Le contrôle de qualité des vaccins, tout le long de la chaîne de distribution qui va de la Pharmacie vétérinaire au vaccinateur, a permis de surveiller les deux campagnes de vaccination et d'apprécier parrallèlement la qualité de la chaîne du froid : congélateurs et réfrigérateurs en état de marche, glacières toujours pleines de glace et bonne conduite du vaccinateur.

Au terme de ces deux années de campagne de vaccination du cheptel bovin (et petits ruminants), il est possible d'affirmer que l'animal ivoirien ayant subi les deux campagnes a reçu, à chaque séance, une dose de bonne qualité. Cette qualité de la vaccination a été prouvée par la sérosurveillance de la PB en 1990 et en 1991 : l'analyse d'un échantillon de sérums représentatif du cheptel bovin national a en effet révélé un taux corrigé de 82,35 p. 100 de couverture immunitaire après la première campagne de 1989 et un taux corrigé de 88,26 p. 100 après la vaccination de 1990 (2). Toutefois, malgré la bonne conservation des vaccins utilisés en Côte-d'lvoire, des chutes de titre ont été enregistrées. Aussi faut-il espérer que les recherches actuelles pour la mise au point d'un vaccin thermostable permettront rapidement la mise sur le marché d'un produit plus facile à utiliser. II est évident qu'un allègement des conditions de conservation sous froid entraînerait une diminution très importante des coûts des campagnes de prophylaxie. Un tel contrôle des campagnes de vaccination (titrages, chaîne du froid, sérologie postvaccinale) est fort coûteux mais demeure indispensable. Il doit être placé sous la responsabilité du laboratoire et dirigé par une équipe indépendante des équipes de vaccination.

\section{Remerciements}

Nous tenons à remercier le projet PARC de Côte-d'lvoire pour son soutien sur le plan financier, MM. BONY et SANOGO pour leur aide technique, l'ensemble des agents des laboratoires de pathologie animale et des projets SODEPRA et le Dr K.M. KOUASSI, responsable de la Pharmacie vétérinaire.

COUACY-HYMANN (E.), KODJO (A.), OUATTARA (M.), KANGA (K.), DIAWARA (S.), DOMENECH (J.). Control of the quality of cold chain and vaccines during the National vaccination campaigns. Revue Élev. Méd. vét. Pays trop., 1992, 45 (2) : 129-133

During the two vaccination campaigns against rinderpest and contagious bovine pleuropneumonia (CBPP) in 1989 and 1990, 507 vaccine samples were collecled for quality control at each step of the distribution chain from the Veterinary Pharmacy of Abidjan to the vaccinator on the field. Parallel to that, the quality of the cold chain was checked. A total of 463 titrations were performed. All the titration levels were above the OIE/FAO standards, i.e. $10^{2.5} \mathrm{DICT}_{50} / \mathrm{ml}$ for rinderpest Valence and $10^{7}$ viable germs $/ \mathrm{ml}$ for contagious bovine pleuropneumonia Vaience. Key words : Cattle Vaccine - Rinderpest - Contagious bovine pleuropneumonia - Refrigerated storage - Refrigerated transport - Côte-d'Ivoire. 


\section{Communication}

\section{Bibliographie}

1. COUACY-HYMANN (E.). Rapport d'activité. Service de virologie et de contröle de qualité des vaccins. Bingerville, Laboratoire de pathologie animale, 1988

2. COUACY-HYMANN (E.), KODJO (A.), DIAWARA (S.), DOMENECH (J.). Contrôle de l'immunité postvaccinale antibovipestique après les campagnes de vaccination de 1989 et 1990 en Côte-d'Ivoire. Revue Elev. Méd vét Pays trop. 1991, 44 (4) : 415-421.

3. DOMENECH (J.), HOSTE (C.), VIGIER (M.). Conservalion du vaccin antipéripneumonique lyophilisé, souche $\mathrm{KH}_{3} \mathrm{~J} \mathrm{Sr}+$. Revue Élev. Méd. vét. Pays trop., 1976, 29 (4) : 295-304.

4. MAN (J.C.)(DE). The probability of Most Probable Numbers. Eur. J. appl. Microbiol., 1975, $1: 67-68$.

5. PALYA (V.), RWEYEMAMU (M.M.). Standard Operating Procedures (S.O.P.) for Rinderpest cell culture vaccine (Live). Addis-Ababa, PANVAC. 1991.84 p. (RAF/88/050)

6. PROVOST (A.), PERREAU (P.), BRÉARD (A.), LE GOFF (C.), MAR TEL (J.L.), COTTEW (G.S.). Péripneumonie contagieuse bovine. Revue sci. tech. Off. int. Epiz., 1987, 6 (3):565-624.

7. SYLLA (D.). Contrôle de qualité du vaccin contre la peste bovine sur culture cellulaire. Dakar, Centre panafricain des vaccins (PANVAC), 1991. 48 p. (Projet CTP RAF/88/050)

8. TAYLOR $(\mathrm{J}$.). The estimation of numbers of bacteria by tenfold dilution series. I appl. Basteriol. 1962, 25 (1) : 54-61

9. THIAUCOURT (F.), DI MARIA (A.). Note sur le titrage des vaccins péripneumoniques. Nouvelle approche d'un vieux problème, essais préliminaires. Revue Elev. Méd. vét. Pays trop., 1989, 42 (3) : 389-391.

10. TULASNE (J.J.). Production et contrôle de qualité du vaccin monovalent contre la péripneumonie contagieuse des bovidés. In : Séminaire FAO du 19-23 sept. 1988, Bamako, Mali.

\section{Le portage asymptomatique de bovins et chèvres Créole guéris de la cowdriose en Guadeloupe}

\section{E. Camus ।* $^{*}$}

CAMUS (E.). Le portage asymptomatique de bovins et chèvres Créole guéris de la cowdriose en Guadeloupe. Revue Élev. Méd. vét. Pays trop., $1992,45(2): 133-135$

Les chèvres et les bovins Créole de Guadeloupe peuvent rester porteurs de cowdriose (Cowdria ruminantium) après la guérison jusqu'à 11 mois pour les chèvres et deux mois pour les bovins. Le portage a été démontré en nourrissant des nymphes d'Amblyomma variegatum sur les animaux guéris puis les tiques adultes qui en sont issues sur des chèvres sensibles. Cow'dria ruminantium n'est pas décelé en permanence dans le sang pendant la durée du portage. Mots clés : Chèvre Créole - Bovin Créole - Cowdriose - Hôte Cowdria ruminantium - Anblyomma variegatum - Guadeloupe.

\section{Introduction}

La cowdriose a été diagnostiquée en 1980 en Guadeloupe (7) et en 1984 dans les îles voisines de Marie-Galante (5) et d'Antigua (3). Elle risque de s'étendre aux autres îles des Antilles, voire au continent américain, par la diffusion de son vecteur, la tique Amblyomma variegatum. L'extension de la cowdriose peut aussi se produire par l'exportation de bovins et de petits ruminants malades ou porteurs asymptomatiques de l'agent Cowdria ruminantium.

1. CIRAD-EMVT, BP 1232, 97185 Poinie-à-Pitre Cedex, Guadeloupe.

* Avec la collaboration technique de R. APRELON et P. THAMS.

Reçu le 12.2.1992, accepté le 25.6.1992.
Dans le cadre d'une éventuelle prochaine campagne d'éradication de la tique $A$. variegatum, il est également important de connaître la persistance maximale de Cowdria chez l'hôte.

La première évaluation de la durée du portage asymptomatique en Guadeloupe indiquait une très faible persistance de Cowdria aussi bien chez la chèvre Créole (2) que chez le bovin Créole (4) : huit jours. Mais le portage était révélé par des larves d'A. variegatum nourries sur des animaux guéris, et gorgées au stade nymphal suivant sur des chèvres sensibles. L'utilisation d'un modèle nymphe-adulte d'A. hebraeum au Zimbabwe (1) mettait en évidence un portage beaucoup plus long : 223,246 et 161 jours, respectivement pour le mouton, le bovin et le buffle africain.

II devenait alors important de vérifier si l'utilisation de nymphes d'A. variegatum gorgées sur des animaux guéris de cowdriose, puis nourries sur des chèvres sensibles, permettait de révéler un portage asymptomatique plus long qu'avec le système larve-nymphe ; c'est l'objectif de ce travail.

\section{Matériel et méthode}

Trois chèvres et trois génisses zébus, toutes de race Créole de Guadeloupe, âgées respectivement de un an et de huit mois, maintenues jusque-là sans contact avec la cowdriose, sont infectées expérimentalement :

- les trois chèvres par inoculation intraveineuse de $2 \mathrm{ml}$ de sang infecté avec le stock Gardel de Cowdria ;

les trois génisses avec 40 nymphes infectées au stade larvaire sur une chèvre inoculée avec le stock Gardel.

La température rectale des six ruminants est observée tous les matins. Dès qu'elle dépasse $40^{\circ} \mathrm{C}$, les trois chèvres sont traitées avec de l'oxytétracycline puis subissent une infection virulente homologue un mois après pour vérifier qu'elles ont bien été infectées. Les trois génisses Créole ne sont pas traitées ni réinfectées. Un test sérologique par immunofluorescence indirecte (stock Kümm), est pratiqué avant l'infection sur les six animaux pour contrôler leur virginité vis-à-vis de la cowdriose. Un mois après le challenge pour les chèvres, un à deux mois après la primo-infection pour les génisses, une quarantaine de nymphes à jeun sont nourries sur les six animaux. Cette installation est répétée une fois par mois pendant 6 à 12 mois.

Les nymphes gorgées, une fois récoltées, sont mises à muer dans des incubateurs où l'hygrométrie est maintenue à $95 \mathrm{p}$. 100 et la température entre 22 et $26^{\circ} \mathrm{C}$.

Environ un mois après chaque installation, chaque lot de tiques adultes provenant des nymphes est nourri sur une chèvre sensible à la cowdriose : d'abord les mâles, puis les femelles trois à six jours après. 